\title{
An illuminated respiratory activity monitoring system identifies priming-active compounds in plant seedlings
}

\author{
Judith Loogen ${ }^{1}$, André Müller ${ }^{2,3}$, Arne Balzer ${ }^{2,3}$, Sophie Weber ${ }^{4}$, Kathrin Schmitz ${ }^{2,3}$, Roxanne Krug ${ }^{5}$, \\ Ulrich Schaffrath ${ }^{2,3}$, Jörg Pietruszk ${ }^{2,5,6}$, Uwe Conrath ${ }^{2,3}$ and Jochen Büchs ${ }^{1,2^{*}}$
}

\begin{abstract}
Background: Growing large crop monocultures and heavily using pesticides enhances the evolution of pesticideinsensitive pests and pathogens. To reduce pesticide use in crop cultivation, the application of priming-active compounds (PrimACs) is a welcome alternative. PrimACs strengthen the plant immune system and could thus help to protect plants with lower amounts of pesticides. PrimACs can be identified, for example, by their capacity to enhance the respiratory activity of parsley cells in culture as determined by the oxygen transfer rate (OTR) using the respiration activity monitoring system (RAMOS) or its miniaturized version, $\mu$ RAMOS. The latter was designed for with suspensions of bacteria and yeast cells in microtiter plates (MTPS). So far, RAMOS or $\mu$ RAMOS have not been applied to adult plants or seedlings, which would overcome the limitation of ( $\mu$ )RAMOS to plant suspension cell cultures.
\end{abstract}

Results: In this work, we introduce a modified $\mu$ RAMOS for analysis of plant seedlings. The novel device allows illuminating the seedlings and records the respiratory activity in each well of a 48-well MTP. To validate the suitability of the setup for identifying novel PrimAC in Arabidopsis thaliana, seedlings were grown in MTP for seven days and treated with the known PrimAC salicylic acid (SA; positive control) and the PrimAC candidate methyl 1-(3,4-dihydroxyphenyl)2-oxocyclopentane-1-carboxylate (Tyr020). Twenty-eight h after treatment, the seedlings were elicited with flg22, a 22-amino acid peptide of bacterial flagellin. Upon elicitation, the respiratory activity was monitored. The evaluation of the OTR course reveals TyrO20 as a likely PrimAC. The priming-inducing activity of TyrO20 was confirmed using molecular biological analyses in A. thaliana seedlings.

Conclusion: We disclose the suitability of $\mu R A M O S$ for identifying PrimACs in plant seedlings. The difference in OTR during a night period between primed and unprimed plants was distinguishable after elicitation with flg22. Thus, it has been shown that the $\mu R A M O S$ device can be used for a reliable screening for PrimACs in plant seedlings.

Keywords: Sustainable agriculture, Oxygen transfer rate, Respiratory activity, Defense priming-inducing chemistry, Plant protection, Plant immunity

\footnotetext{
*Correspondence: jochen.buechs@avt.rwth-aachen.de

${ }^{1}$ AVT.BioVT, RWTH Aachen University, Forckenbeckstraße 51,

52074 Aachen, Germany

Full list of author information is available at the end of the article
}

\begin{abstract}
Background
As the world population continuously grows, there is an increased need for food, feed, fibers, and bioenergy [29]. These stocks are mainly derived from crops, such as corn, rice or wheat, that are usually grown in large monocultures. These are heavily sensitive to biotic and abiotic stress. Synthetic pesticides are widely used to effectively fight pests and pathogens. However, they can
\end{abstract}


accumulate in the soil or plant and, thus, they raise ecological and health concerns. In addition, the heavy use of pesticides provokes the emergence of pests and pathogens with insensitivities to the chemicals [29]. To avoid the risks that comes with synthetic pesticides, exploiting the plant immune system emerged as a supportive, or even alternative, approach for eco-friendly plant protection [8].

Upon attack by pathogens or after treatment with certain chemicals, plants can develop resistance or enhance their resistance to further pathogen attack [3]. This phenomenon is referred to as induced resistance which is frequently associated with defense priming [8]. The term refers to the enhanced capacity of plant cells to activate defense responses. Priming-associated induced resistance responses include systemic acquired resistance (SAR) [11]. SAR is a broad-spectrum immune response induced by salicylic acid (SA) and N-hydroxypipecolic acid $[15,33]$. Thus, upon treatment with N-hydroxypipecolic acid, SA or substances with a similar mode of action, plant cells are frequently primed for the enhanced activation of defense responses [20, 42 , and this usually comes with the establishment of SAR $[8,11]$. Defense priming, whether induced by SA, treatment with another chemical compound, or previous pathogen attack frequently enhances the capacity of the plant also to ward off abiotic stresses, such as salinity, drought, flooding, chilling, or heat [18, 34, 41]. Until now, one common method to identify PrimACs is measuring the accumulation of mRNA transcripts of WRKY6 and WRKY53 [19]. WRKY6 and WRKY53 are reliable marker genes for defense priming in A. thaliana [19]. The accumulation of mRNA transcripts of these genes is not much induced during priming [19]. However, the accumulation of WRKY6/53 transcripts induced by low doses of flg22 is more robust in primed than unprimed plants [19]. It has been demonstrated that priming involves considerably fewer costs and is hardly prone to pest or pathogen adaptation [26, 44]. Thus, triggering priming by eco-friendly chemicals represents a promising means for sustainable pest and disease control. However, identifying priming-inducing chemistry is difficult and applicable screening systems for priming activity are rare. Conrath and associates developed [20] and recently optimized [36] a mid- throughput screen for compounds that prime microbial pattern-induced furanocoumarin secretion in parsley cell suspension cultures. In addition, using a respiratory activity monitoring system (RAMOS), Schilling et al. [37] showed that their property can identify priming-active compounds (PrimACs) to enhance the respiratory activity, as measured by the oxygen transfer rate (OTR) of a parsley cell culture $[1,2]$. In this study, the word respiration comprises the sum of the physical and biochemical processes in an organism by which oxygen is conveyed to tissues and cells. Two possible explanations for the enhanced oxygen consumption during priming have been previously discussed. One explanation claimed that the activation of mitochondrial alternative oxidase (AOX) might cause enhanced oxygen consumption [13, $14,28,32]$. The other explanation suggested that the NADPH oxidase caused the enhanced oxygen consumption by the synthesis of reactive oxygen species $[6,13$, $21]$. Both explanations are conceivable separately or in combination.

For enhanced throughput, a $\mu$ RAMOS device has been developed that utilizes parley suspension cells in 48-well microtiter plates (MTPs) [16]. The RAMOS technology repeatedly switches between phases of gassed and sealed bioreactors applying specific valves. During the sealed phase, the oxygen partial pressure, measured via a respective sensor, decreases due to respiration of the cultivated organisms [1]. The OTR from the gas to the aqueous phase is proportional to the determined slope of the oxygen partial pressure during the sealed phases [1, 16]. The $\mu$ RAMOS is based on standard 48-well MTPs. It uses the same measurement principle as the RAMOS technology but is provided with another valve and sensor technology [16]. Whilst the RAMOS is equipped with electrochemical sensors, the $\mu$ RAMOS uses immobilized fluorescence sensor spots to measure the oxygen partial pressure [16].

Both RAMOS and $\mu$ RAMOS have been optimized for use with parsley cells in culture $[16,38,40]$. To overcome the limitations of $(\mu)$ RAMOS to parsley cell suspensions, in this work, a protocol for the use of intact plants in the $\mu$ RAMOS device is developed. With the experimental setup, a light-emitting diode (LED) module is introduced that enables each well of a 48-well MTP to be individually irradiated with white light to ensure synchronized growth [5]. In this study, we present the setup for a LED- $\mu$ RAMOS combination and a standard procedure for the identification of new PrimACs on plant seedlings. For a proof-of-principle of the introduced LED- $\mu$ RAMOS combination and the standard procedure, a model screening for PrimACs has been performed using the newly synthesized methyl 1-(3,4-dihydroxyphenyl)-2-oxocyclopentane-1-carboxylate (Tyr020) as model test compound, which has been introduced by Krug et al. [23]. The power of the novel devise has been shown using SA as a positive control and spotting Tyr020 as a novel PrimAC in A. thaliana. Tyr020 was validated as a PrimAC using molecular biological analyses [10]. 


\section{Results}

\section{LED module- $\mu$ RAMOS combination}

A LED module was developed to enable the simultaneous growth of seeds. It enables adjusted light condition for germination and growth if needed, as it is the case for $A$. thaliana seedlings. The plant seedlings are grown in each well of a 48-well MTP in controlled light condition. The module consists of 48 individually dimmable LEDs (Lumitronix) mounted on a plate of the size of a commercial 48-well MTP (Fig. 1A). Optical isolators ensure that the light cannot enter neighboring wells, provided a black, clear-bottomed MTP is applied on top of the LED module. Because all wells are irradiated by an individual LED, equal conditions are maintained for each well, regardless of the well position. The $\mu$ RAMOS device is applied atop the MTP (Fig. 1B) and enables a measurement of the respiration activity in each plate well. Figure $1 \mathrm{C}$ displays a photograph of the setup with $A$. thaliana seedlings on top of the LED module with the attached $\mu$ RAMOS device. For illustration purposes, a translucent MTP has been drawn in this. This newly introduced combination of LED module and $\mu$ RAMOS (LED- $\mu$ RAMOS) is the basis of the presented protocol to identify new PrimACs.

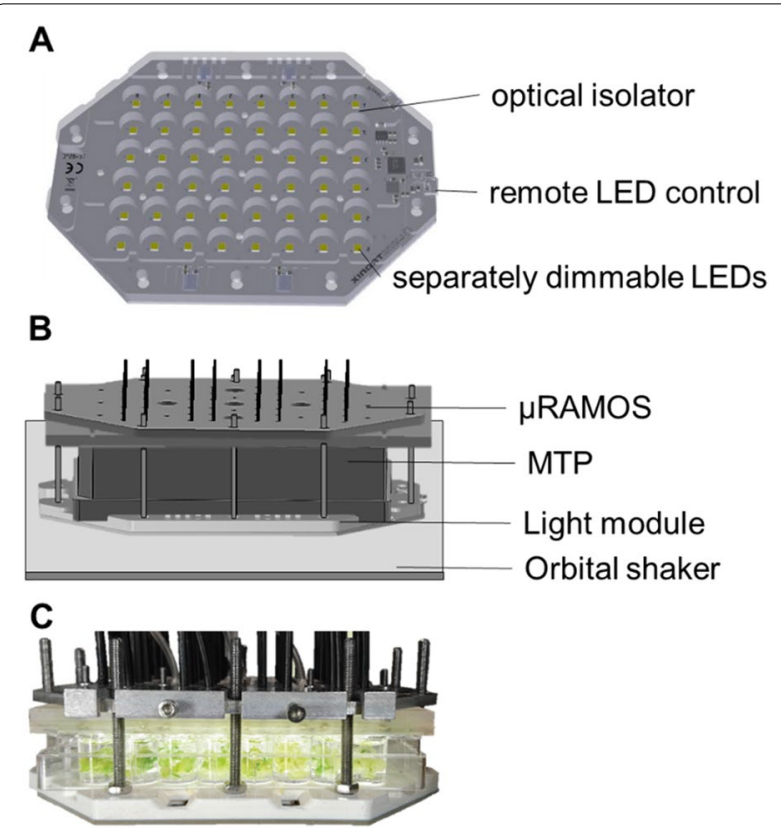

Fig. 1 A Custom-made LED module. 48 individually dimmable LEDs for each well of a MTP. An optical isolator prevents interference between individual wells of the MTP. B Combination of the LED module with a $\mu R A M O S$ device. The LED module is mounted below the MTP. MRAMOS measures the partial pressure of oxygen in each well and calculates the resulting OTR. C Photograph of the assembled device with A. thaliana plant seedlings. Translucent MTPs were used for illustration purposes. For online monitoring, black MTPs with transparent bottoms were used

\section{Standard procedure to screen for PrimACs in plants using MRAMOS}

We developed a standard procedure that enables the search for PrimACs in the introduced LED- $\mu$ RAMOS combination (Fig. 2). A. thaliana seeds were surface sterilized with $70 \%(\mathrm{v} / \mathrm{v})$ ethanol (Fig. 2A). Using a seed dispenser, sterilized seeds were individually transferred into the wells of an MTP (Fig. 2B). Sterile MS medium was added to each well (Fig. 2C). To ensure synchronous and effective germination of seeds, the MTP was kept for $24 \mathrm{~h}$ at $4{ }^{\circ} \mathrm{C}$. Then, the MTPs were incubated for 3 weeks at $20{ }^{\circ} \mathrm{C}$. During incubation, a $16 \mathrm{~h}$ day/ $8 \mathrm{~h}$ night cycle was applied using the LED module (Fig. 2C). After those 3 weeks, the seedlings were treated with SA or Tyr020 (Fig. 2D) and incubated for another $28 \mathrm{~h}$ in growth condition. Then, seedlings were sprayed with 50 pM flg22. After elicitation, the respiratory activity of the seedlings was measured for $45 \mathrm{~h}$ by applying the $\mu$ RAMOS (Fig. 2D) atop of the MTP.

\section{OTR of $A$. thaliana seedlings grown in the LED- $\mu$ RAMOS combination}

To validate the suitability of the novel LED- $\mu$ RAMOS device for measuring the respiratory activity of $A$. thaliana seedlings, seedlings were grown in a MTP in the above described conditions in the LED- $\mu$ RAMOS devise for $168 \mathrm{~h}$. The OTR was monitored during the $16 \mathrm{~h}$ day/8 $\mathrm{h}$ night period. The course of the OTR over the $168 \mathrm{~h}$ period revealed that during the day, oxygen was produced as indicated by negative OTR values (Fig. 3). During the night, low amounts of oxygen were consumed, as obvious by positive OTR values (Fig. 3). However, the quantities of oxygen consumed were rather low, as obvious by the OTR being close to $0 \mathrm{mmol} / \mathrm{L} / \mathrm{h}$ for most of the time in the night period, indicating starvation. At the end of the fourth day period, $500 \mu \mathrm{L}$ of sterile MS medium were added to each well to avoid desiccation of the seedlings. This stimulated increased oxygen consumption during the following night period, as seen in Fig. 3 at 80 h. After supplementation with MS medium, the oxygen produced during the following day periods was lower than at the day periods before. Based on these results, it could be recognized that $2.5 \mathrm{~g} / \mathrm{L}$ sucrose is not sufficient for a measurement to identify PrimACs. Thus, the MS medium was supplemented with $20 \mathrm{~g} / \mathrm{L}$ sucrose to ensure that the seedlings are not starving. With this change, it is secured that the OTR remains above $0 \mathrm{mmol} / \mathrm{L} / \mathrm{h}$ for an elongated night period, which is applied for the following PrimAC identification measurement. Positive OTR values are necessary to measure the influence of PrimACs on the respiration activity of the seedlings. 
(A) Sterilization of seeds
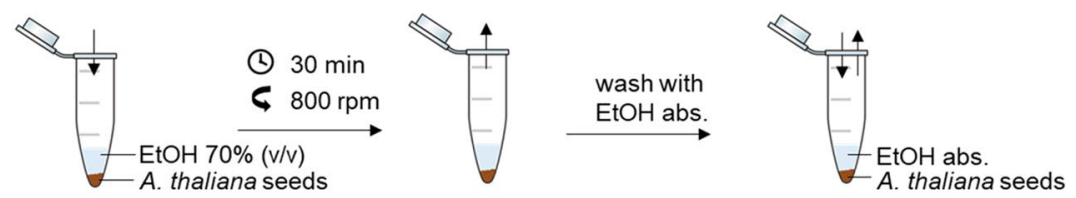

(B) Distribution of seeds to MTPS

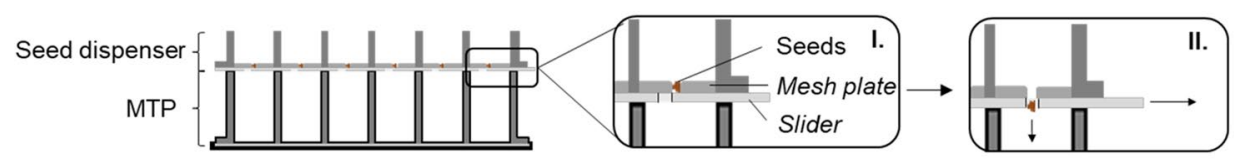

\section{(C) Cultivation in MTPs}

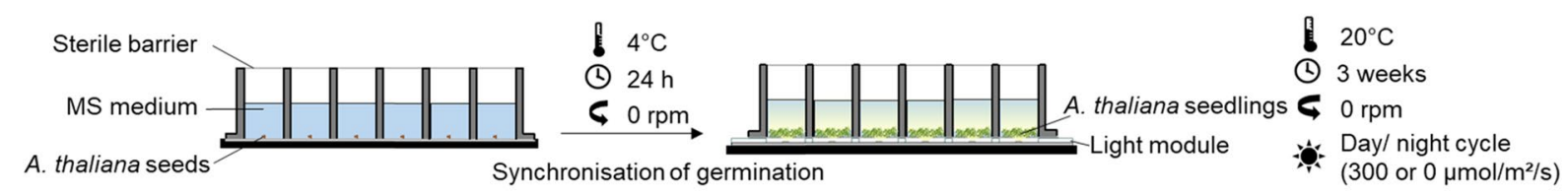

\section{(D) Priming measurement}

At $-28 \mathrm{~h}$ addition of priming-active compound

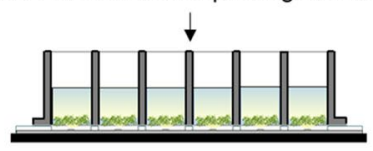

f $20^{\circ} \mathrm{C}$

(1) $28 \mathrm{~h}$

$600 \mathrm{rpm}$

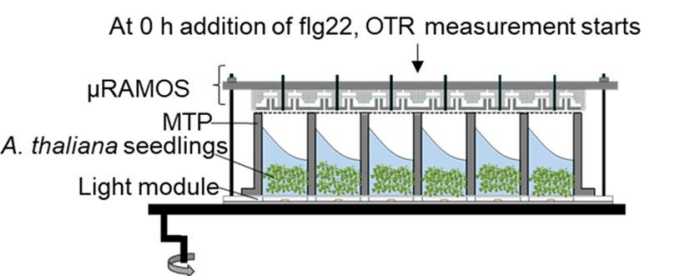

$20^{\circ} \mathrm{C}$

() $72 \mathrm{~h}$ measurement time

$660 \mathrm{rpm}$

Night cycle $\left(0 \mu \mathrm{mol} / \mathrm{m}^{2} / \mathrm{s}\right)$

Fig. 2 Scheme of the setup for PrimAC identification using A. thaliana seedlings in the LED- $\mu$ RAMOS device. A Seeds were sterilized by washing with 70\% (v/v) ethanol and ethanol absolute (EtOH abs.). B A seed dispenser was used to transfer sterilized seeds to the wells of a MTP. C Cultivation in MTPs for the effective and synchronized germination of seeds. A. thaliana was grown on $1.5 \mathrm{~mL}$ MS medium for 3 weeks at a 16-h day/8-h night cycle. D The respiratory activity was measured in the absence of light at $20^{\circ} \mathrm{C}$ and $600 \mathrm{rpm}$ shaking frequency with a shaking diameter of $3 \mathrm{~mm}$. Treatments with compounds was done $28 \mathrm{~h}$ before the OTR measurement were started. Seedlings were elicited using $50 \mathrm{pM}$ flg $22 \mathrm{at}$ time zero ( $0 \mathrm{~h}$ )

\section{Measurement of priming-inducting activity}

The screening for PrimACs was performed as illustrated in Fig. 2. A. thaliana seedlings were diveded in six groups and, where appropriate, treated at $-28 \mathrm{~h}$ before elicitation with flg22 at time point $0 \mathrm{~h}$. Immediately after elicitation, the OTR measurement was started (Fig. 4). In the first hour of recording, all measurements show significantly increased OTR values. These are various runningin phenomena of the $\mu$ RAMOS device, for example, the MTP needs to first reach the temperature of the system before a stable OTR value can be measured. This period of the first hour is, therefore, irrelevant for the screening of PrimACs and is not further discussed. Twelve wells of the MTP were neither pretreated nor elicited to obtain a negative control (Fig. 4). The course of the OTR of the negative control remained at a low level $(\sim 1 \mathrm{mmol} / \mathrm{L} / \mathrm{h})$. Seedlings in 5 wells were treated with $100 \mu \mathrm{M}$ SA and seedlings in 3 wells supplemented with $25 \mu \mathrm{M}$ Tyr020 at $-28 \mathrm{~h}$. These 8 wells were not supplied with $50 \mathrm{pM}$ flg22. This we did to record the course of the OTR of pretreated but unelicited plants (Fig. 4). The OTR curve of this group remained at $\sim 1 \mathrm{mmol} / \mathrm{L} / \mathrm{h}$, comparable to the negative control.

The course of the OTR (red and blue dotted) curves of these seedlings were like those of the negative control (black graph, Fig. 4). Furthermore, seedlings in 5 wells were not pretreated but elicited with $50 \mathrm{pM}$ flg22 (green curve). After elicitation for $8 \mathrm{~h}$, the OTR of the seedlings was significantly higher than that of the negative control. The OTR of the unprimed but elicited seedlings remained high for $40 \mathrm{~h}$. Seedlings in 3 wells were treated with $100 \mu \mathrm{M}$ SA (red curve, positive control) or $25 \mu \mathrm{M}$ Tyr020 (blue curve) at $-28 \mathrm{~h}$ before the OTR measurement was being started. These seedlings were elicited with $50 \mathrm{pM}$ flg22 at the $0 \mathrm{~h}$ time point. The OTR values of SA-pretreated and later flg22-elicited seedlings were much higher than those of unprimed but elicited seedlings. The course of the OTR of Tyr020-treated and elicited seedlings was like the course of the OTR of the positive control. Thus, 


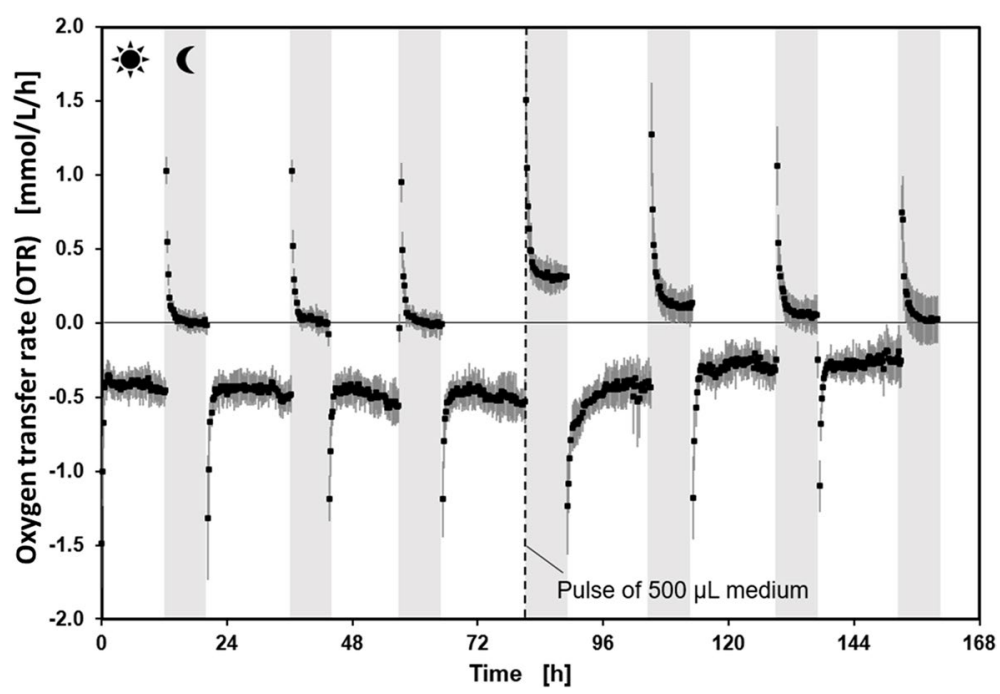

Fig. 3 OTR of A. thaliana seedlings during $16 \mathrm{~h}$ day/8 $\mathrm{h}$ night cycle in the LED- $\mu$ RAMOS combination. The white and grey background illustrate day and night periods, respectively, and are indicated by sun or moon. Three-week old A. thaliana seedlings, grown as in Fig. 2C, were transferred to the LED- $\mu$ RAMOS combination and kept at $600 \mathrm{rpm}$ shaking frequency, $3 \mathrm{~mm}$ shaking diameter, $20^{\circ} \mathrm{C}$, in $1.5 \mathrm{~mL} \mathrm{MS}$ medium with $2.5 \mathrm{~g} / \mathrm{L}$ sucrose, initial pH 5.7, light module irradiation intensity $300 \mu \mathrm{mol} / \mathrm{m}^{2} / \mathrm{s}$ at day time, $0 \mu \mathrm{mol} / \mathrm{m}^{2} / \mathrm{s}$ at night time. The standard deviation of 12 replicates is represented by the grey shadow around the data points

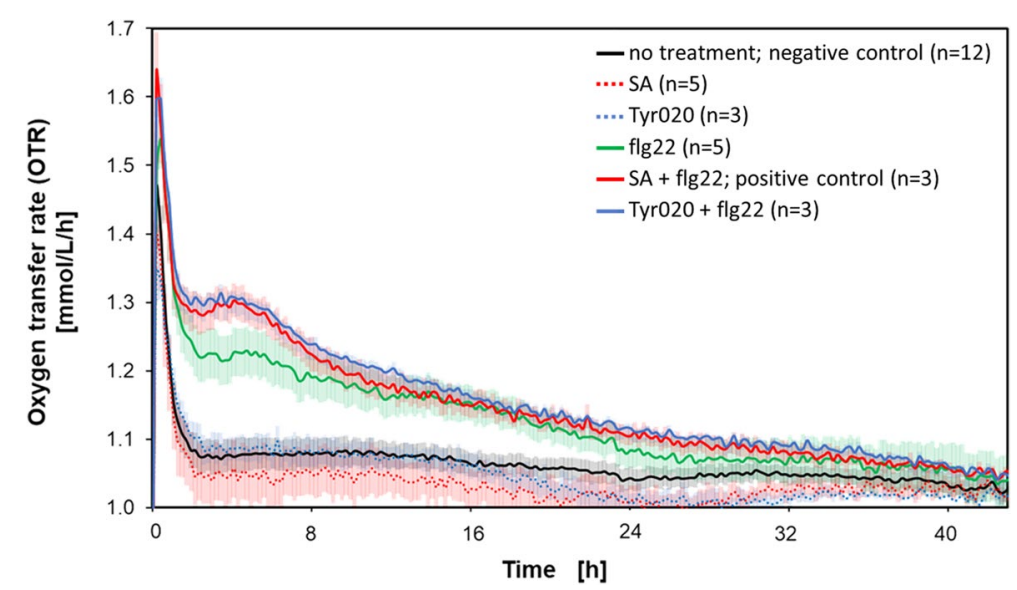

Fig. 4 Respiratory activity of A. thaliana seedlings after treatment with priming compounds with and without flg22 elicitation, and reference cultivation without treatment with chemicals. OTR as a function of time of A. thaliana seedlings primed with $100 \mu \mathrm{M}$ SA and 50 pM flg22 (red) or $25 \mu \mathrm{M}$ Tyr020 and 50 pM flg22 (blue). Reference cultivations: without additives, negative control (black), addition of $100 \mu \mathrm{M}$ SA only (red dotted line), $25 \mu \mathrm{M}$ Tyr020 (blue dotted line), and with the addition of $50 \mathrm{pM}$ flg22 only (green line). SA and Tyr020 were added at the -28 $\mathrm{h}$ time point relative to the time point of flg22 addition ( $0 \mathrm{~h}$ ). Three-week-old $A$. thaliana seedlings were transferred to the $\mu$ RAMOS device, according to Fig. $2 \mathrm{D}$, and cultivated at $600 \mathrm{rpm}$ shaking frequency, $3 \mathrm{~mm}$ shaking diameter, filling volume $1.5 \mathrm{~mL}, 20^{\circ} \mathrm{C}$, in MS medium with $20 \mathrm{~g} / \mathrm{L}$ sucrose, initial pH 5.7 . The number of replica ( $n$ ) is specified for each condition in the legend. The standard deviation of replicates is indicated by the shadow surrounding the data

Tyr020 was identified as promising PrimAC candidate in A. thaliana.

To verify or disprove Tyr020 as a PrimAC, we investigated whether the compound would induce known molecular mechanisms of defense priming in A. thaliana
$[8,9]$. To do so, we determined the accumulation of mRNA transcript of the WRKY6 and WRKY53 transcription factor genes inappropriately treated plants. To investigate whether Tyr020 would prime $A$. thaliana for the enhanced accumulation of WRKY6/53 mRNA 
transcript, soil-grown $A$. thaliana seedlings were divided into six groups, each of six plants. One group was treated with a wettable powder (WP; negative control) which serves as a wetting agent for S-methyl benzo[d][1,2,3] thiadiazole-7-carbothioate (BTH). The second group was treated with a WP formulation of BTH (positive control), which is a functional SA analog Friedrich et al. [15, 24] that strongly triggers defense priming in A. thaliana $[19,22]$. The four remaining groups of seedlings were treated with different concentrations of Tyr020. Fortyeight hours after treatment, three seedlings of each group were elicited with flg22. The remaining three seedlings of each group remained unelicited. Three hours after flg22 elicitation, seedlings were harvested and analyzed for the accumulation of mRNA transcript of WRKY6 and WRKY53 by qRT-PCR. As shown in Fig. 5A, the flg22-elicited seedlings of the WP group showed a small increase in the accumulation of WRKY6 and WRKY53 mRNA transcript compared to the unelicited seedlings. The unelicited group of BTH-treated seedlings showed a similar extent of mRNA transcript accumulation of the two WRKY genes (Fig. 5). When compared to the WP group of seedlings, the flg22-elicited group of BTH-pretreated seedlings displayed a significantly higher accumulation of WRKY6 and WRKY53 transcript (Fig. 5A). This result confirms the defense priming-inducing activity of BTH in A. thaliana seedlings and verifies that the experimental setup allows the detection of priming-inducing activity. The Tyr020-treated but unelicited seedlings accumulated WRKY6 and WRKY53 mRNA transcript to an extent like the unelicited WP group (Fig. 5A). When compared to the WP groups, the Tyr020 pretreated and later elicited group of seedlings had an increased level of mRNA transcript of both these genes. Only at $100 \mu \mathrm{M}$ Tyr020, pretreated plants showed a significant $(p \leq 0.05)$ increase in the accumulation of WRKY6 and WRKY53 mRNA transcript when compared to the WP group. The groups of seedlings that were treated with 10 or $50 \mu \mathrm{M}$ Tyr020 only for one of the WRKY genes showed a significant increase in mRNA transcript levels (Fig. 5A).
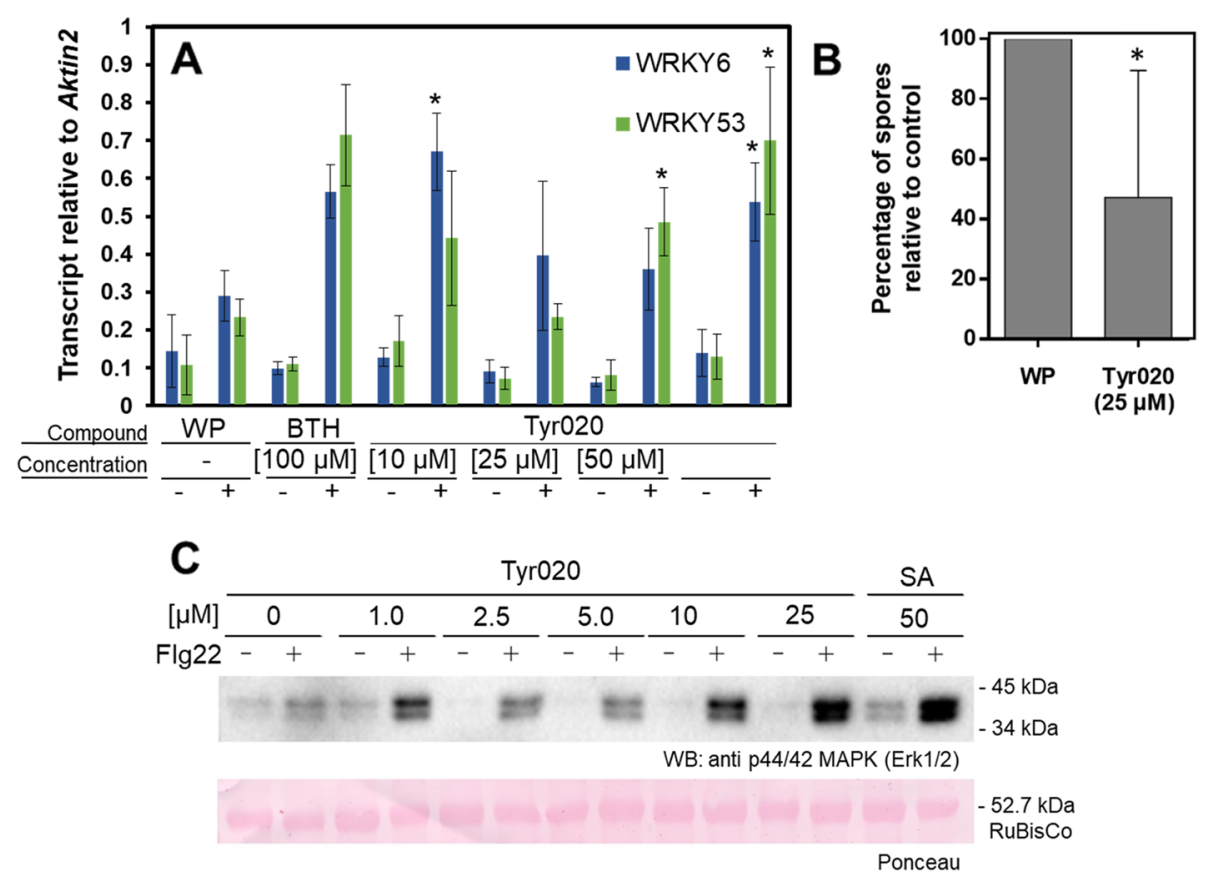

Fig. 5 Verification of identified priming compound in soil grown A. thaliana plants. A Tyr020 primes A. thaliana plants for enhanced WRKY 6 and WRKY53 defense gene activation. Six-week-old plants remained untreated (-) or sprayed with wettable powder (+) or the indicated concentration of Tyr020 in wettable powder (WP) (+) before they were elicited (+) or not (-) with flg22. As positive control $100 \mu M$ Benzothiadiozole (BTH) was used. The accumulation of WRKY6 and WRKY53 mRNA transcript was determined by qRT-PCR. Stars (*) indicate significant differences between control $(\mathrm{WP} ;+)$ and sample $(p \leq 0.05)$. B Sulforaphane (SFN) reduces downy mildew disease. Plants were treated with WP or a WP formulation of Tyr020 $(25 \mu \mathrm{M})$. Twenty-four hours later, we spray-inoculated plants with a suspension of Hyaloperonospora arabidopsidis (Hpa) conidiospores $\left(4 \times 10^{4}\right.$ spores per $\mathrm{mL}$ of water). Inoculated plants were kept at high humidity in short day. After $7 \mathrm{~d}$, we determined the number of spores released by Hpa. Data were analyzed by Student's $t$ test. Asterisk denotes a statistically significant difference with $95 \%$ confidence. Data presented are means \pm SD. $n>5$. C A. thaliana seedlings pretreated with Tyr020 have enhanced phosphorylation of mitogen-activated protein kinase (MPK) activation motifs upon flg22 challenge. This indicates stronger MPK activation in Tyr020-pretreated plants. As positive control A. thaliana seedlings were treated with $50 \mu \mathrm{M}$ salicylic acid (SA) 
However, in sum, the results of this experiment disclose the priming-inducing capacity of Tyr020 at the level of defense gene expression, and they reveal that Tyr020 seems to poise WRKY6/53 for enhanced transcription upon further challenge.

To investigate whether Tyr020 would affect the infection of A. thaliana by the oomycete pathogen Hyaloperonospora arabidopsidis (Hpa), A. thaliana (Col-0) plants were sprayed with a wettable powder (WP) formulation of Tyr020 before they were inoculated with Hpa. The Noco race of $\mathrm{Hpa}$ causes downy mildew disease on A. thaliana Col-0 plants (Coates and Beynon [12]). Figure $5 \mathrm{~B}$ shows that pretreatment with Tyr020 seems to reduce the susceptibility of $A$. thaliana to downy mildew disease, as obvious by reduced Hpa sporulation on Tyr020-pretreated plants. This suggests that Tyr020 activates defense priming and reduces the susceptibility to Hpa infection in A. thaliana.

To further investigate Tyr020's mode of action, the accumulation of phosphorylated mitogen-activated protein kinases (MPKs) was analyzed in A. thaliana seedlings upon adequate treatments. Beckers et al. [4] demonstrated that in this plant defense priming is associated with enhanced levels of latent MPK3 and MPK6. However, these enzymes remained catalytically inactive until further stimulation of the plants [4]. Once challenged, more MPK molecules became phosphorylationactivated in primed compared to unprimed plants [4]. To investigate the phosphorylation of MPKs in Tyr020treated $A$. thaliana seedlings, appropriately treated seedlings were subjected to western blotting analysis and immunodetection with phospho-motif-specific antibodies (Fig. 5C). Two untreated seedlings were used as a negative control, whereas two other seedlings were treated with $50 \mu \mathrm{M}$ SA (positive control) to activate defense priming. Ten seedlings were treated with Tyr020 at concentrations from 1 to $25 \mu \mathrm{M}$. One seedling of each condition was later elicited with flg22. As shown in Fig. 5C, MPK phosphorylation was detected in all seedlings elicited with flg22. For the unpretreated but elicited seedling, the phosphorylation signal was very low compared to the elicited positive control. No or only weak MPK phosphorylation was found in all the unelicited samples. Except for $1 \mu \mathrm{M}$, Tyr020 with increasing concentration strongly enhanced the flg22-induced MPK phosphorylation signal that, at $25 \mu \mathrm{M}$, was almost as strong as the phosphorylation signal in seedlings that were primed with $50 \mu \mathrm{M}$ SA and later challenged with flg22 (Fig. 5C). This result supports the above conclusions from the $\mu$ RAMOS and gene expression studies, claiming Tyr020 as a previously unknown PrimAC.

\section{Discussion}

An LED module was developed that allows equal light exposure of plant seedlings in each well of a 48-well MTP. Development of a similar illumination device has been published previously [27]. The described resulting improvements of individually illuminated plant cultivation could be reproduced with the introduced LED module. The first measurement (Fig. 3) with the LED$\mu$ RAMOS combination showed that MS medium supplemented with $2.5 \mathrm{~g} / \mathrm{L}$ sucrose was not sufficient to measure respiration activity of the seedlings during the night period, as indicated by OTR values close to $0 \mathrm{mmol} /$ $\mathrm{L} / \mathrm{h}$. Schilling et al. have shown that PrimACs enhance the respiration activity of plant cells [37]. Therefore, for an application of the LED- $\mu$ RAMOS combination for the successful identification of PrimACs, measurable respiration of the seedlings is required. The problem that due to starvation, no respiration activity was measured at night could be overcome by the addition of fresh MS medium. The addition of fresh medium to A. thaliana in the LED$\mu$ RAMOS leads to an increased OTR during the following night cycle, showing the increased metabolic activity of the plant seedlings due to the newly added nutrients. The MS medium supplemented with sucrose seems to satisfy the seedlings' carbon demand and, therefore, the photorespiration might have become lower, as it was already published for sugarcane by Lobo et al. [25]. In the second and third night cycle after addition of fresh medium, the OTR was still higher than before addition. In summary, the addition of sterile medium was successful so that in the future, longer cultivation of plant seedlings is also conceivable. In order to reduce the risk of possible contamination by the addition of fresh medium and to minimize the handling steps, it was decided to increase the initial concentration of the supplemented sucrose from $2.5 \mathrm{~g} / \mathrm{L}$ to $20 \mathrm{~g} / \mathrm{L}$.

This result suggested that metabolic changes of the plant seedlings seem to be more promoted during the night cycle. As previous studies on plant suspension cell cultures already showed, the respiration activity gives crucial information about their metabolism [17, 31, 43].

Hence, the evaluation of candidates of PrimACs is best executed during an elongated night period. The growth was performed applying a $8 \mathrm{~h}$ day $/ 16 \mathrm{~h}$ night cycle, using the LED module. The beginning of the elongated night period was only associated with the addition of flg22. At the same time, the $\mu$ RAMOS device was mounted on top of the MTP, and the OTR measurement started. After elicitation, primed plant seedlings showed much higher OTR values than unprimed, but elicited, or only elicited plant seedlings (Fig. 4). Thus, PrimAC enhance the capacity of respiratory activity of intact plants. This enhanced capacity can be measured 
with the LED- $\mu$ RAMOS combination, but other oxygen-based measurement systems might also be conceivable. The measurement systems published by Scafaro et al. [35], or O'Leary et al. [30], both relying on the Q2 oxygen sensor (Astec Global, The Netherlands), measure the oxygen consumption of leaves and roots. Nevertheless, both measurement systems could potentially be used for PrimAC identification. As this enhanced capacity of respiratory activity is just measured at this stage of experimental protocol, the $\mu$ RAMOS device needs to be applied, while the germination, growth and priming can be performed separately in a microtiter plate. Through time-shifted initiation of cultivations in microtiter plates, the screening of 48 plant seedlings every $40 \mathrm{~h}$ per $\mu$ RAMOS device becomes possible. The results of the model screening (Fig. 4) indicate that it might be possible to reduce this measurement period to $8 \mathrm{~h}$ since the difference between successfully primed plant seedlings and the corresponding negative control is most evident in the first $8 \mathrm{~h}$ after elicitation. A shortened measurement period would further enhance the throughput of the screening method to up to 48 plant seedlings per $8 \mathrm{~h}$ per $\mu$ RAMOS device.

\section{Conclusions}

In this paper, the use of the $\mu$ RAMOS for intact plant seedlings in 48-well MTP was demonstrated. The major advantage of using intact plants rather than cell suspension cultures is the differentiation status and overcoming the limitations of parsley and some other plant species. $\mu$ RAMOS is likely compatible with essentially any crop and, thus, closer to application. However, in contrast to soil-grown plants, measurements can be done at early growth stages in controlled conditions, as parameters like nutrition and light become adjustable. By switching from the RAMOS device, which allows simultaneous screenings in eight shake flasks, to the $\mu$ RAMOS device, which enables 48 simultaneous screenings, the throughput per experiment was drastically increased. Based on the increased OTR after elicitation (Fig. 4), Tyr020 has been identified as previously unknown PrimAC.

The newly introduced LED- $\mu$ RAMOS combination enables individual light intensities and irradiation periods for each well, while the OTR is independently measured online. This allows for different irradiation intensities, which might be required by different plant species within one experiment. In the future, candidates for PrimACs and combinations thereof could simultaneously be tested for their priming-inducing activity in different crops.

\section{Methods}

\section{Chemicals}

SA, BTH and wettable powder are commercially available and Tyr020 has been synthesized according to Krug et al. [23]. Krug et al. published this compound under the name compound 8a [23]. SA, BTH, Tyr020 and wettable powder were diluted in distilled water according to the indicated concentrations. No additional solvent was used.

\section{LED module}

The LED module is custom-made by Lumitronix ${ }^{\circledR}$ and consists of 48 individual dimmable LEDs (Fig. 1). The plate can be mounted underneath a transluminescentbottomed 48-well MTP resulting in one LED beneath each well. To allow a light gradient within the plate, each LED is individually adjustable. Optical isolators prevent stray light from LEDs into neighboring wells. The OTR was determined in shake flasks using the RAMOS $[1,2]$ in MTPs using the $\mu$ RAMOS. Both devices were developed and built in house [16, 38]. Measurements were performed using black, clearbottom microtiter plates. The $\mu$ RAMOS device was mounted on top of the MTP. The measurement was performed at $20^{\circ} \mathrm{C}$ with a shaking frequency of $600 \mathrm{rpm}$ and a shaking diameter of $3 \mathrm{~mm}$. The filling volume was $1.6 \mathrm{~mL}$ per well. The OTR was calculated based on measured partial pressure of oxygen and registered settings, including the headspace volume. This calculation was adjusted to changing headspace volumes, i.e., after the addition of PrimACs or fresh medium.

\section{A. thaliana growth}

Arabidopsis seeds (Arabidopsis thaliana, accession Col0 ) were purchased from the European Arabidopsis Stock Centre (Nottingham Arabidopsis Stock Centre; NASC). The seeds were washed in $70 \%(\mathrm{v} / \mathrm{v})$ ethanol for $30 \mathrm{~min}$ and then twice in $100 \%(\mathrm{v} / \mathrm{v})$ ethanol for $1 \mathrm{~min}$, according to Fig. 2. Dried seeds were transferred to a MTP using a seed dispenser to a number of $15 \pm 5$ seeds per well. The seeds were provided with $1.5 \mathrm{~mL}$ (MS) medium. MS medium, including vitamins (M0222; Duchefa Biochemie B.V.), was prepared as recommended by the manufacturer and supplemented with $2.5 \mathrm{~g} / \mathrm{L}$ sucrose. The $\mathrm{pH}$ value was adjusted to 5.7 using $0.01 \mathrm{M}$ potassium hydroxide, before the medium was autoclaved $\left(121^{\circ} \mathrm{C}, 20 \mathrm{~min}\right)$.

For stratification the seed-loaded MTPs were stored at $4{ }^{\circ} \mathrm{C}$ overnight to ensure synchronous and efficient germination [5]. On the next days, the plates were cultivated on the LED module at $20^{\circ} \mathrm{C}$. After 3 weeks, the $\mu$ RAMOS device was mounted atop. 
Cultivation, priming and elicitation of soil-grown $A$. thaliana seedlings

A. thaliana (accession Col-0; NASC) seedlings were grown on soil with a $16 \mathrm{~h}$ day/ $8 \mathrm{~h}$ night cycle applied in a pests-free room. When needed, the soil was irrigated. After 4 - 6 weeks, seedlings were used for conventional priming experiments. The leaves of $A$. thaliana seedlings were sprayed with Tyr020 dilutions in ascending concentrations between $1 \mu \mathrm{M}$ and $100 \mu \mathrm{M}$. As positive control, the leaves of another plant seedling were sprayed with $100 \mu \mathrm{M}$ BTH. As negative control, leaves of plant seedlings were sprayed with $21 \%(\mathrm{w} / \mathrm{v})$ wettable powder in water. Every condition was applied on six plant seedlings each. The plant seedlings were further grown for $48 \mathrm{~h}$, before three plants of each condition were infiltrated with $1 \mathrm{nM}$ flg22 solution.

\section{Determination of WRKY6, WRKY53 and Actin2 expression}

Five hours after elicitation, leaves were harvested and homogenized. RNA was isolated from frozen leaves using the TRIZOL method, as published by [7]. The RT-qPCR reactions were performed in a $10 \mu \mathrm{L}$ volume. The PCR mixture was consisting of $2.7 \mu \mathrm{L}$ nuclease-free water, $5 \mu \mathrm{l}$ Takyon TM No Rox SYBR MasterMix dTTP Blue (Eurogentec), $0.15 \mu \mathrm{L}$ forward primer, $0.15 \mu \mathrm{L}$ reverse primer, and $2.5 \mu \mathrm{L}$ template. The according primers are listed in Table 1. The amplification was performed using a PCR machine (Applied Biosystems). The PCR was performed for $3 \mathrm{~min}$ at $95{ }^{\circ} \mathrm{C}$ as initial denaturation, followed by 40 cycles of $15 \mathrm{~s}$ at $95^{\circ} \mathrm{C}$ for denaturation, $60 \mathrm{~s}$ at $60^{\circ} \mathrm{C}$ as annealing, $15 \mathrm{~s}$ at $95^{\circ} \mathrm{C}$ for extension. The final extension was set to $60^{\circ} \mathrm{C}$ for $1 \mathrm{~min}$.

\section{SDS-PAGE, western-blotting analysis and immunodetection}

To detect MPK phosphorylation sites, leaves of the soilgrown seedlings were harvested and homogenized at $5 \mathrm{~h}$ after elicitation. Frozen tissue was either ground using the Precellys 24 homogenizer (Bertin Instruments). All tubes and buffers were kept cold during the procedure. Ground plant material was washed twice with $100 \%$ acetone and centrifuged at $16,100 \mathrm{~g}$ at $4{ }^{\circ} \mathrm{C}$ for $5 \mathrm{~min}$. The pellet was resuspended in 10\% (w/v) TCA in

Table 1 Primers used for RT-qPCR

\begin{tabular}{ll}
\hline Primer & Sequence $\mathbf{5}^{\prime} \mathbf{- 3}^{\prime}$ \\
\hline WRKY6 & ACTTCACGGTCATTATCTCCAGC \\
WRKY53 & TGAATTAGGTTCCGGTGATC \\
& CTCCATCGGCAAACTCTTCAC \\
Actin2 & CCGAGCGTACAACTTATTCCG \\
& GGTAACATTGTGCTCAGTGGTGG \\
& GGTGCAACGACCTTAATCTTCAT \\
\hline
\end{tabular}

acetone and were transferred to an ultrasound ice-water bath for $10 \mathrm{~min}$ followed by another centrifugation step $\left(16,100 \mathrm{~g}, 4{ }^{\circ} \mathrm{C}, 5 \mathrm{~min}\right)$. Then, the pellet was washed once with $10 \%(\mathrm{w} / \mathrm{v})$ TCA in acetone, once with $10 \%$ TCA (w/v) and once with $80 \%(\mathrm{v} / \mathrm{v})$ acetone. The supernatant was discarded and the pellet was resuspended at RT in freshly prepared dense SDS buffer (100 mM Tris$\mathrm{HCl} \mathrm{pH} \mathrm{8.0,} \mathrm{30 \%} \mathrm{(w/v)} \mathrm{sucrose,} \mathrm{2 \%} \mathrm{(w/v)} \mathrm{SDS,} \mathrm{5 \%} \mathrm{(v/v)}$ $\beta$-mercaptoethanol). An equal volume of phenol/Tris$\mathrm{HCl} \mathrm{pH} 8.0$ (Applichem) was added to each tube. Samples were centrifuged at $16,100 \mathrm{~g}$ at RT for $20 \mathrm{~min}$. The upper phase of each tube was split into two new tubes, 5 volumes of $100 \mathrm{mM}$ ammonium acetate in methanol were added, and total protein was precipitated at $-20{ }^{\circ} \mathrm{C}$ for $60 \mathrm{~min}$. Proteins were collected by centrifugation at $16,100 \mathrm{~g}$ at $4{ }^{\circ} \mathrm{C}$ for $5 \mathrm{~min}$. Pellets were washed once with $100 \mathrm{mM}$ ammonium acetate in methanol and once with $80 \%(\mathrm{v} / \mathrm{v})$ acetone, and the pellets were dried. To determine total protein concentration, pellets were resuspended in buffer $(7.7 \mathrm{M}$ urea, $2 \mathrm{M}$ thiourea, $300 \mathrm{mM} \mathrm{NaCl}, 0.25 \%$ (w/v) CHAPS, $50 \mathrm{mM} \mathrm{NaH}_{2} \mathrm{PO}_{4}$ $\mathrm{pH} 8,50 \mathrm{mM}$ Tris $\mathrm{pH} 8,20 \mathrm{mM}$ imidazole) and incubated at RT for $1 \mathrm{~h}$ before Bradford protein assay (Quick-Start Bradford; BioRad) was performed. Protein samples were resuspended in loading buffer (10X Sample Reducing Agent, 4X LDS Sample Buffer; NuPAGE) to equal concentrations and heated at $95{ }^{\circ} \mathrm{C}$ for $10 \mathrm{~min}$. The denatured samples were applied to a two parted polyacrylamide gel, consisting of a $4 \%$ collection gel and a $12 \%$ separation gel. The pockets were loaded with $5 \mu \mathrm{L}$ sample, and gel electrophoresis was performed at $175 \mathrm{~V}$ for $60 \mathrm{~min}$. MES, pH 7.3, was used as running buffer.

For the subsequent western blot, the proteins were electrophoretically transferred from the SDS gel to a nitrocellulosemembrane (Carl Roth) applying $60 \mathrm{~min}$ at $250 \mathrm{~mA}$. After blotting, the membrane was washed 2 times for 5 min each in TBST buffer $(20 \mathrm{mM}$ tris; $150 \mathrm{mM} \mathrm{NaCl} ; 0.1 \%$ (v/v) Tween 20). The blocking was performed for $60 \mathrm{~min}$ in a $5 \%(\mathrm{w} / \mathrm{v})$ skimmed milk powder (Nutricia Protifar) in TBST buffer solution. After blocking, the membrane was washed 2 times for $5 \mathrm{~min}$ each in TBST buffer. The primary antibody anti p44/42 (Cell SignalingTechnologyR) was diluted 1:1000 in a 5\% (w/v) Bovine Serum Albumin (Panreac AppliChem) in TBST buffer solution. The membrane was incubated for $12 \mathrm{~h}$ at $10{ }^{\circ} \mathrm{C}$. After incubation, the membrane was washed twice for $5 \mathrm{~min}$ in TBST buffer.

As secondary antibody, the anti-rabbit IgG, HRP-linked Antibody \#7074 (Cell SignalingTechnologyR) was applied. It was diluted 1:2000 in a solution of $5 \%(\mathrm{w} / \mathrm{v})$ skimmed milk powder in TBST buffer solution. The membrane was incubated for $1 \mathrm{~h}$ at room temperature in the solution before being washed twice in TBST for each $5 \mathrm{~min}$. 
For protein detection, the ChemiDocTMMP Imagine System (BioRadR) with white light irradiation was used.

\section{Ponceau staining}

To check for equal protein loading, the membrane was stained with Ponceau S. For staining, the Ponceau S solution (Panreac AppliChem) was used, and the producer's protocol was followed precisely.

\section{Determining A. thaliana susceptibility to Hyaloperonospora arabidopsidis ( $\mathrm{Hpa})$}

A. thaliana Col-0 plants were grown in short-day $(8 \mathrm{~h}$ light of $120 \mu \mathrm{mol} / \mathrm{m}^{2} / \mathrm{s}^{1}, 22{ }^{\circ} \mathrm{C}, 65 \%$ relative humidity). Two-week-old plants were sprayed with wettable powder (WP) (control) or a WP formulation of Tyr020 (final concentration $25 \mu \mathrm{M}) .24 \mathrm{~h}$ after treatment, plants were inoculated by spraying with a conidiospore suspension of Hpa (race Noco; $4 \times 10^{4}$ spores per $\mathrm{mL}$ of water). Inoculated plants were covered with a transparent lid to ensure high humidity and kept in short-day condition. After $7 \mathrm{~d}$, the number of spores released by Hpa was determined as described by Schmitz et al. [39].

\section{Acknowledgements}

We thank G.J.M. Beckers for technical advice.

\begin{abstract}
Authors' contributions
All authors contributed to the design of the research; A.M., S.W., A.B. K.S., and R.K. performed the research; all authors analyzed and discussed the data; J.L. drafted the manuscript. J.B. U.C., U.S., and J.P. read, commented, and revised the manuscript. J.B. initiated the study, assisted with data analysis and manuscript preparation. All authors read and approved the final manuscript.
\end{abstract}

\section{Funding}

Open Access funding enabled and organized by Projekt DEAL. The scientific activities of the Bioeconomy Science Center were financially supported by the Ministry of Innovation, Science and Research in the NRW Strategy Project BioSC (No. 313/323-400-00213).

\section{Availability of data and materials}

The datasets generated during and analyzed during the current study are available from the corresponding author on request.

\section{Declarations}

Ethics approval and consent to participate

Not applicable.

\section{Consent for publication}

All authors agree that the present work will be published.

\section{Competing interests}

The authors declare that they have no competing interest.

\section{Author details}

1AVT.BioVT, RWTH Aachen University, Forckenbeckstraße 51, 52074 Aachen, Germany. ${ }^{2}$ Bioeconomy Science Center (BioSC), C/O Research Center Jülich, 52425 Jülich, Germany. ${ }^{3}$ Department of Plant Physiology, RWTH Aachen University, Worringer Weg 1, 52074 Aachen, Germany. ${ }^{4}$ Institute for Bioand Geoscience, IBG-2: Plant Science, Forschungszentrum Jülich, 52425 Jülich, Germany. ${ }^{5}$ Institut Für Bioorganische Chemie (IBOC), Heinrich-Heine-Universität Düsseldorf Im Forschungszentrum Jülich, 52426 Jülich, Germany. ${ }^{6}$ Institut
Für Bio- Und Geowissenschaften, IBG-1: Biotechnologie, Forschungszentrum Jülich, 52425 Jülich, Germany.

Received: 23 December 2020 Accepted: 10 June 2021

Published online: 05 July 2021

\section{References}

1. Anderlei T, Büchs J. Device for sterile online measurement of the oxygen transfer rate in shaking flasks. Biochem Eng J. 2001;7:157-62. https://doi. org/10.1016/S1369-703X(00)00116-9.

2. Anderlei T, Zang W, Papaspyrou M, Büchs J. Online respiration activity measurement (OTR, CTR, RQ) in shake flasks. Biochem Eng J. 2004;17:18794. https://doi.org/10.1016/S1369-703X(03)00181-5.

3. Beckers GJM, Conrath U. Priming for stress resistance: from the lab to the field. Curr Opin Plant Biol. 2007;10:425-31. https://doi.org/10.1016/j.pbi. 2007.06.002.

4. Beckers GJM, Jaskiewicz M, Liu Y, Underwood WR, He SY, Zhang S, Conrath U. Mitogen-activated protein kinases 3 and 6 are required for full priming of stress responses in Arabidopsis thaliana. Plant Cell. 2009;21:944-53. https://doi.org/10.1105/tpc.108.062158.

5. Burghardt LT, Edwards BR, Donohue K. Multiple paths to similar germination behavior in Arabidopsis thaliana. New Phytol. 2016;209:1301-12.

6. Chen Z, Silva H, Klessig D. Active oxygen species in the induction of plant systemic acquired resistance by salicylic acid. Science. 1993;262(5141):1883-6.

7. Chomczynski P. A reagent for the single-step simultaneous isolation of RNA, DNA and proteins from cell and tissue samples. Biotechniques. 1993;15(532-4):536-7.

8. Conrath U, Beckers GJM, Langenbach CJG, Jaskiewicz MR. Priming for enhanced defense. Annu Rev Phytopathol. 2015;53:97-119. https://doi. org/10.1146/annurev-phyto-080614-120132.

9. Conrath U. Molecular aspects of defence priming. Trends Plant Sci. 2011;16:524-31. https://doi.org/10.1016/j.tplants.2011.06.004.

10. Conrath U. Systemic acquired resistance. Plant Signal Behav. 2006;1:17984. https://doi.org/10.4161/psb.1.4.3221.

11. Conrath U, Pieterse CM, Mauch-Mani B. Priming in plant-pathogen interactions. Trends Plant Sci. 2002;7:210-6. https://doi.org/10.1016/ S1360-1385(02)02244-6.

12. Coates ME, Beynon JL. Hyaloperonospora Arabidopsidis as a pathogen model. Annu Rev Phytopathol. 2010;48:329-45. https://doi.org/10.1146/ annurev-phyto-080508-094422.

13. Cvetkovska M, Alber NA, Vanlerberghe GC. The signaling role of amitochondrial superoxide burst during stress. Plant Signal Behav. 2013:8(1):e22749.

14. Cvetkovska M, Vanlerberghe GC. Alternative oxidase impacts the plantresponse to biotic stress by influencing the mitochondrial generation ofreactive oxygen species. Plant Cell Environ. 2013;36(3):721-32.

15. Delaney TP, Uknes S, Vernooij B, Friedrich L, Weymann K, Negrotto D, et al. A Central Role of Salicylic Acid in Plant Disease Resistance. Science. 1994;1247-1250. https://doi.org/10.1126/science.266.5188.1247.

16. Flitsch D, Krabbe S, Ladner T, Beckers M, Schilling J, Mahr S, et al. Respiration activity monitoring system for any individual well of a 48-well microtiter plate. J Biol Eng. 2016;10:14. https://doi.org/10.1186/ s13036-016-0034-3.

17. Geipel K, Socher ML, Haas C, Bley T, Steingroewer J. Growth kinetics of a Helianthus annuus and a Salvia fruticosa suspension cell line: Shake flask cultivations with online monitoring system. Eng Life Sci. 2013;13(6):593-602.

18. Janda T, Szalai G, Tari I, Páldi E. Hydroponic treatment with salicylic acid decreases the effects of chilling injury in maize (Zea mays L.) plants. Planta. 1999;208:175-80. https://doi.org/10.1007/s004250050547.

19. Jaskiewicz M, Conrath U, Peterhänsel C. Chromatin modification acts as a memory for systemic acquired resistance in the plant stress response. EMBO Rep. 2011;12:50-5. https://doi.org/10.1038/embor.2010.186.

20. Kauss H, Theisinger-Hinkel E, Mindermann R, Conrath U. Dichloroisonicotinic and salicylic acid, inducers of systemic acquired resistance, enhance fungal elicitor responses in parsley cells. Plant J. 1992;2:655-60. https:// doi.org/10.1111/j.1365-313X.1992.tb00134.x. 
21. Kauss H, Jeblick W. Pretreatment of parsley suspension cultures with salicylicacid enhances spontaneous and elicited production of H2O2. Plant Physiol. 1995;108(3):1171-8.

22. Kohler A, Schwindling S, Conrath U. Benzothiadiazole-induced priming for potentiated responses to pathogen infection, wounding, and infiltration of water into leaves requires the NPR1/NIM1 gene in Arabidopsis. Plant Physiol. 2002;128:1046-56. https://doi.org/10.1104/pp.010744.

23. Krug R, Schröder D, Gebauer J, Suljić S, Morimoto Y, Fujieda N, et al. Tyrosinases in organic chemistry: a versatile tool for the a-arylation of ß-dicarbonyl compounds. Eur J Org Chem. 2018;2018:1789-96. https:// doi.org/10.1002/ejoc.201800188.

24. Lawton KA, Friedrich L, Hunt M, Weymann K, Delaney T, Kessmann H, et al. Benzothiadiazole induces disease resistance in Arabidopsis by activation of the systemic acquired resistance signal transduction pathway. Plant J. 1996;10:71-82. https://doi.org/10.1046/j.1365-313x.1996.10010071.x.

25. Lobo AKM, de Oliveira MM, Costa Lima Neto M, Caruso Machado E, Vasconcelos Ribeiro R, Albenisio Gomes Silveira J. Exogenous sucrose supply changes sugar metabolism and reduces photosynthesis of sugarcane through the down-regulation of Rubisco abundance and activity. Plant Physiol. 2015;173:113-21.

26. Martinez-Medina A, Flors V, Heil M, Mauch-Mani B, Pieterse CMJ, Pozo MJ, et al. Recognizing plant defense priming. Trends Plant Sci. 2016;21:81822. https://doi.org/10.1016/j.tplants.2016.07.009.

27. Morschett H, Schiprowski D, Müller C, Mertens K, Felden P, Meyer J, et al. Design and validation of a parallelized micro-photobioreactor enabling phototrophic bioprocess development at elevated throughput. Biotechnol Bioeng. 2017;114:122-31. https://doi.org/10.1002/bit.26051.

28. Norman C, Howell KA, Millar AH, Whelan JM, Day DA. Salicylic acid is anuncoupler and inhibitor of mitochondrial electron transport. Plant Physiol. 2004;134(1):492-501.

29. Oerke E. Crop losses to pests. J Agric Sci. 2006;144:31-43. https://doi.org/ 10.1017/S0021859605005708.

30. O'Leary BM, Oh GGK, Lee CP, Millar AH. Metabolite regulatory interactions control plant respiratory metabolism via target of rapamycin (TOR) kinase activation. Plant Cell. 2020;32(3):666-82.

31. Raval KN, Hellwig S, Prakash G, Ramos-Plasencia A, Srivastava A, Büchs J. Necessity of a two-stage process for the production of azadirachtinrelated limonoids in suspension cultures of Azadirachta indica. J Biosci Bioeng. 2003;96(1):16-22.

32. Rhoads DM, Mclntosh L. Cytochrome and alternative pathway respiration intobacco (effects of salicylic acid). Plant Physiol. 1993;103(3):877-83.

33. Ryals JA, Neuenschwander UH, Willits MG, Molina A, Steiner HY, Hunt MD. Systemic acquired resistance. Plant Cell. 1996;8:1809-19. https://doi.org/ 10.1105/tpc.8.10.1809.
34. Savvides A, Ali S, Tester M, Fotopoulos V. Chemical priming of plants against multiple abiotic stresses: mission possible? Trends Plant Sci. 2016;21:329-40.

35. Scafaro AP, Negrini ACA, O'Leary B, et al. The combination of gas-phase fluorophore technology and automation to enable high-throughput analysis of plant respiration. Plant Methods. 2017;13:16. https://doi.org/ 10.1186/s13007-017-0169-3.

36. Schillheim B, Jansen I, Baum S, Beesley A, Bolm C, Conrath U. Sulforaphane modifies histone $\mathrm{H} 3$, unpacks chromatin, and primes defense. Plant Physiol. 2018;176:2395-405. https://doi.org/10.1104/pp.17.00124.

37. Schilling JV, Mahr S, Schillheim B, Conrath U, Büchs J. Scale down of parsley cell cultures to screen putative priming compounds for agro-industry. New Biotechnol. 2016;33:S38. https://doi.org/10.1016/j.nbt.2016.06.856.

38. Schilling JV, Schillheim B, Mahr S, Reufer Y, Sanjoyo S, Conrath U, Büchs J. Oxygen transfer rate identifies priming compounds in parsley cells. BMC Plant Biol. 2015;15:282. https://doi.org/10.1186/s12870-015-0666-3.

39. Schmitz G, Reinhold T, Göbel C, Feussner I, Neuhaus HE, Conrath U. Limitation of nocturnal ATP import into chloroplasts seems to affect hormonal crosstalk, prime defense, and enhance disease resistance in Arabidopsis thaliana. Mol Plant Microbe Interact. 2010;23:1584-91. https://doi.org/10.1094/MPMI-02-10-0045.

40. Schulte A, Schilling JV, Nolten J, Korona A, Krömke H, Vennekötter J, et al. Parallel online determination of ethylene release rate by shaken parsley cell cultures using a modified RAMOS device. BMC Plant Biol. 2018;18:101. https://doi.org/10.1186/s12870-018-1305-6.

41. Senaratna T, Touchell D, Bunn E, Dixon K. Acetyl salicylic acid (Aspirin) and salicylic acid induce multiple stress tolerance in bean and tomato plants. Plant Growth Regul. 2000;30:157-61. https://doi.org/10.1023/A:10063 86800974.

42. Thulke $\mathrm{O}$, Conrath $U$. Salicylic acid has a dual role in the activation of defence-related genes in parsley. Plant J. 1998;14:35-42. https://doi.org/ 10.1046/j.1365-313X.1998.00093.x.

43. Ullisch DA, Müller CA, Maibaum S, Kirchhoff J, Schiermeyer A, Schillberg S, et al. Comprehensive characterization of two different Nicotiana tabacum cell lines leads to doubled GFP and HA protein production by media optimization. J Biosci Bioeng. 2012;113(2):242-8.

44. van Hulten M, Pelser M, van Loon LC, Pieterse CMJ, Ton J. Costs and benefits of priming for defense in Arabidopsis. Proc Natl Acad Sci U S A. 2006;103:5602-7.

\section{Publisher's Note}

Springer Nature remains neutral with regard to jurisdictional claims in published maps and institutional affiliations.
Ready to submit your research? Choose BMC and benefit from:

- fast, convenient online submission

- thorough peer review by experienced researchers in your field

- rapid publication on acceptance

- support for research data, including large and complex data types

- gold Open Access which fosters wider collaboration and increased citations

- maximum visibility for your research: over 100M website views per year

At BMC, research is always in progress.

Learn more biomedcentral.com/submissions 